PROCEEDINGS OF THE AMERICAN MATHEMATICAL SOCIETY

Volume 126, Number 7, July 1998, Pages 1885-1896

S 0002-9939(98)04421-9

\title{
ONCE MORE NICE EQUATIONS FOR NICE GROUPS
}

\author{
SHREERAM S. ABHYANKAR AND PAUL A. LOOMIS
}

(Communicated by Ronald M. Solomon)

\begin{abstract}
In a previous paper, nice quintinomial equations were given for unramified coverings of the affine line in nonzero characteristic $p$ with the projective symplectic isometry group $\operatorname{PSp}(2 m, q)$ and the (vectorial) symplectic isometry group $\operatorname{Sp}(2 m, q)$ as Galois groups where $m>2$ is any integer and $q>1$ is any power of $p$. Here we deform these equations to get nice quintinomial equations for unramified coverings of the once punctured affine line in characteristic $p$ with the projective symplectic similitude group $\operatorname{PGSp}(2 m, q)$ and the (vectorial) symplectic similitude group $\operatorname{GSp}(2 m, q)$ as Galois groups.
\end{abstract}

\section{INTRODUCTION}

Let $m>2$ be any integer, let $q>1$ be any power of a prime $p$, and consider the polynomial $F=F(Y)=Y^{n}+T^{q} Y^{u}+X Y^{v}+T Y^{w}+1$ in indeterminates $T, X, Y$ over a field $k$ of characteristic $p$, where $n=1+q+\cdots+q^{2 m-1}$, $u=1+q+\cdots+q^{m}, v=1+q+\cdots+q^{m-1}, w=1+q+\cdots+q^{m-2}$, and consider its Galois group $\operatorname{Gal}(F, k(X, T))$ and the Galois group $\operatorname{Gal}(\Phi, k(X, T))$ of its subvectorial associate $\Phi=\Phi(Y)=F\left(Y^{q-1}\right)$. Also consider the deformation $F^{\sharp}=F^{\sharp}(Y)=$ $Y^{n}+T^{q} Y^{u}+X Y^{v}+S^{v-w} T Y^{w}+S^{v}$ of $F$ together with its subvectorial associate $\Phi^{\sharp}=\Phi^{\sharp}(Y)=F^{\sharp}\left(Y^{q-1}\right)$, where $S$ is another indeterminate, and their Galois $\operatorname{groups} \operatorname{Gal}\left(F^{\sharp}, k(X, S, T)\right)$ and $\operatorname{Gal}\left(\Phi^{\sharp}, k(X, S, T)\right)$. In the "More Nice Equations" paper [A06] it was shown that if $k$ is algebraically closed, then $\operatorname{Gal}(F, k(X, T))=$ the projective symplectic isometry group $\operatorname{PSp}(2 m, q)$ and $\operatorname{Gal}(\Phi, k(X, T))=$ the (vectorial) symplectic isometry group $\operatorname{Sp}(2 m, q) .{ }^{1}$ By modifying the proof given in [A06], we shall show that if $\operatorname{GF}(q) \subset k$, then $\operatorname{Gal}\left(F^{\sharp}, k(X, S, T)\right)=$ the projective symplectic similitude group $\operatorname{PGSp}(2 m, q)$ and $\operatorname{Gal}\left(\Phi^{\sharp}, k(X, S, T)\right)=$ the (vectorial) symplectic similitude group $\operatorname{GSp}(2 m, q){ }^{2}$ The said proof given in [A06], as well as the proofs of the corresponding unitary and orthogonal group equations given in

Received by the editors December 1, 1996.

1991 Mathematics Subject Classification. Primary 12F10, 14H30, 20D06, 20 E22.

The first author's work was partly supported by NSA grant MDA 904-97-1-0010, and the second author's work was partly supported by a PRF grant at Purdue University.

${ }^{1}$ Here we regard $\operatorname{Sp}(2 m, q)$ as acting on nonzero vectors. For the vectorial associate $\widehat{\Phi}(Y)=$ $Y \Phi(Y)$ we then have $\operatorname{Gal}(\widehat{\Phi}, k(X, T))=\mathrm{Sp}(2 m, q)$ regarded as acting on the entire vector space $\operatorname{GF}(q)^{2 m}$. For generalities about the Galois groups of projective, subvectorial, and vectorial polynomials see [A09].

${ }^{2}$ Again here we regard $\operatorname{GSp}(2 m, q)$ as acting on nonzero vectors. For the vectorial associate $\widehat{\Phi}^{\sharp}(Y)=Y \Phi^{\sharp}(Y)$ we then have $\operatorname{Gal}\left(\widehat{\Phi}^{\sharp}, k(X, S, T)\right)=\operatorname{GSp}(2 m, q)$ regarded as acting on the entire vector space $\operatorname{GF}(q)^{2 m}$. 
[A05] and [A07] respectively, involved very intricate factorizations for some multivariate polynomials. At the end of [A08] these factorizations were codified into a Mantra. By invoking this Mantra, we shall give here a very short and transparent derivation for the factorization of [A06] and its generalizations needed for the GSp equations.

As a by-product of the present modified proof, we shall show that the above results about the Galois groups of $\Phi$ and $F$ continue to hold when we replace the assumption of $k$ being algebraically closed by the weaker assumption that $\mathrm{GF}(q) \subset k$. As another by-product of the present modified proof, we shall show that if $\operatorname{GF}(q) \subset k$, then, for every divisor $d$ of $q-1$, upon letting $\Phi^{(d)}$ be obtained by substituting $S^{d}$ for $S$ in $\Phi^{\sharp}$ we have $\operatorname{Gal}\left(\Phi^{(d)}, k(X, S, T)\right)=\operatorname{GSp}^{(d)}(2 m, q)$ where we define $\operatorname{GSp}^{(d)}(2 m, q)$ by the condition that $\operatorname{Sp}(2 m, q) \triangleleft \mathrm{GSp}^{(d)}(2 m, q) \triangleleft \mathrm{GSp}(2 m, q)$ with $\operatorname{GSp}(2 m, q) / \operatorname{GSp}^{(d)}(2 m, q)=Z_{d}{ }^{3}$ and upon letting $F^{(d)}$ be obtained by substituting $S^{d}$ for $S$ in $F^{\sharp}$ we have $\operatorname{Gal}\left(F^{(d)}, k(X, S, T)\right)=\operatorname{PGSp}^{(d)}(2 m, q)$ where we define $\operatorname{PGSp}^{(d)}(2 m, q)=$ the image of $\operatorname{GSp}^{(d)}(2 m, q)$ under the canonical epimorphism of $\operatorname{GL}(2 m, q)$ onto $\operatorname{PGL}(2 m, q)$, and we note that then $\operatorname{PGSp}^{(d)}(2 m, q)=\operatorname{PSp}(2 m, q)$ or $\operatorname{PGSp}(2 m, q)$ according as $d$ is even or odd. ${ }^{4}$ As noted in [A06], the polynomials $\Phi$ and $F$ are specializations of more general polynomials $\phi_{e}$ and $f_{e}$ whose Galois groups are $\operatorname{Sp}(2 m, q)$ and $\operatorname{PSp}(2 m, q)$ respectively, and which are special cases of the families of polynomials giving unramified coverings of the affine line in characteristic $p$ written down in [A02]. In Section 2 we shall formulate the corresponding more general deformations $\phi_{e}^{\sharp}, \phi_{e}^{(d)}, f_{e}^{\sharp}, f_{e}^{(d)}$ whose Galois groups, under certain conditions, will turn out to be $\operatorname{GSp}(2 m, q), \operatorname{GSp}^{(d)}(2 m, q), \operatorname{PGSp}(2 m, q)$, $\operatorname{PGSp}^{(d)}(2 m, q)$ respectively, and which may be regarded as giving unramified coverings of the once punctured affine line.

In addition to factorization, as in [A03] to [A07], here the basic techniques of calculating Galois groups will be MTR (= the Method of Throwing away Roots) and RTG (= Recognition Theorems for Groups). On the RTG side we shall again use Kantor's characterization of Rank 3 groups in terms of their subdegrees [Kan], supplemented by the Cameron-Kantor Theorem IV [CaK] on antiflag transitive collineation groups. Note that Kantor's Rank 3 characterization depends on the Buekenhout-Shult characterization of polar spaces [BuS] which itself depends on Tits' classification of spherical buildings [Tit]. Recall that the Rank of a transitive permutation group is the number of orbits of its 1-point stabilizer and the sizes of these orbits are called subdegrees. It is a pleasure to thank Nick Inglis and Ganesh Sundaram for stimulating conversations concerning the material of this paper.

\section{Notation AND OUtLine}

Let $k_{p}$ be a field of characteristic $p>0$, let $q>1$ be any power of $p$, and let $m>0$ be any integer. ${ }^{5}$ To abbreviate frequently occurring expressions, for every

\footnotetext{
${ }^{3}$ Since $\operatorname{Sp}(2 m, q) \triangleleft \operatorname{GSp}(2 m, q)$ with $\operatorname{GSp}(2 m, q) / \operatorname{Sp}(2 m, q)=Z_{q-1}$ (see 2.1.2, 2.1.B and 2.1.C of $[\mathrm{KLi}])$, this uniquely characterizes the intermediate group $\operatorname{GSp}^{(d)}(2 m, q)$. Note that, as usual, $<$ and $\triangleleft$ denote subgroup and normal subgroup respectively, and $Z_{d}$ denotes a cyclic group of order $d$.

${ }^{4}$ In view of the previous footnote, this follows from the fact that $\operatorname{PGSp}(2 m, q) / \operatorname{PSp}(2 m, q)=Z_{2}$ or $Z_{1}$ according as $q$ is odd or even (see 2.1.D of [KLi]). Note that if $q$ is even, then $\operatorname{PSp}(2 m, q)=$ $\operatorname{PGSp}(2 m, q)$.

${ }^{5}$ In the Abstract and the Introduction we assumed $m>2$. But in the rest of the paper, unless stated otherwise, we only assume $m>0$.
} 
integer $i \geq-1$ we put

$$
\langle i\rangle=1+q+q^{2}+\cdots+q^{i} \quad(\text { convention: }\langle 0\rangle=1 \text { and }\langle-1\rangle=0) .
$$

Let

$$
f^{b}=f^{b}(Y)=S^{r(m)} X Y^{\langle m-1\rangle}+\sum_{i=1}^{m}\left(S^{r(m+i)} T_{i}^{q^{i}} Y^{\langle m-1+i\rangle}+S^{r(m-i)} T_{i} Y^{\langle m-1-i\rangle}\right)
$$

where $r=(r(0), \ldots, r(2 m))$ is a sequence of nonnegative integers with

$$
r(2 m)=0
$$

such that for some nonnegative integer $t$ we have

$$
q^{i} r(m-i)=r(m+i)+t q^{m}\langle i-1\rangle \quad \text { for } 0 \leq i \leq m
$$

and note that then $f^{b}$ is a polynomial of degree $\langle 2 m-1\rangle=1+q+q^{2}+\cdots+q^{2 m-1}$ in $Y$ with coefficients in the polynomial ring $k_{p}\left[X, S, T_{1}, \ldots, T_{m}\right]$ and in it the coefficient of the highest $Y$-degree term is $T_{m}^{q^{m}}$. Let $\phi^{b}$ and $\widehat{\phi}^{b}$ be the subvectorial and vectorial associates of $f^{b}$ respectively, i.e., let

$$
\begin{aligned}
\phi^{b} & =\phi^{b}(Y)=f^{b}\left(Y^{q-1}\right) \\
& =S^{r(m)} X Y^{q^{m}-1}+\sum_{i=1}^{m}\left(S^{r(m+i)} T_{i}^{q^{i}} Y^{q^{m+i}-1}+S^{r(m-i)} T_{i} Y^{q^{m-i}-1}\right)
\end{aligned}
$$

and

$$
\begin{aligned}
\widehat{\phi}^{b} & =\widehat{\phi}^{b}(Y)=Y \phi^{b}(Y) \\
& =S^{r(m)} X Y^{q^{m}}+\sum_{i=1}^{m}\left(S^{r(m+i)} T_{i}^{q^{i}} Y^{q^{m+i}}+S^{r(m-i)} T_{i} Y^{q^{m-i}}\right)
\end{aligned}
$$

For $0 \leq e \leq m-1$, let $f_{e}^{\sharp}$ be obtained by putting $T_{m}=1$ and $T_{i}=0$ for $e<i<m$ in $f^{b}$, i.e., let

$$
\begin{aligned}
f_{e}^{\sharp}=f_{e}^{\sharp}(Y)= & Y^{\langle 2 m-1\rangle}+S^{r(0)}+S^{r(m)} X Y^{\langle m-1\rangle} \\
& +\sum_{i=1}^{e}\left(S^{r(m+i)} T_{i}^{q^{i}} Y^{\langle m-1+i\rangle}+S^{r(m-i)} T_{i} Y^{\langle m-1-i\rangle}\right)
\end{aligned}
$$

and note that then $f_{e}^{\sharp}$ is a monic polynomial of degree $\langle 2 m-1\rangle=1+q+q^{2}+\cdots+$ $q^{2 m-1}$ in $Y$ with coefficients in the polynomial ring $k_{p}\left[X, S, T_{1}, \ldots, T_{e}\right]$. Now the constant term of $f_{e}^{\sharp}$ is $S^{r(0)}$ and the $Y$-exponent of every other term in $f_{e}^{\sharp}$ is 1 modulo $p$, and hence $f_{e}^{\sharp}-Y f_{e Y}^{\sharp}=S^{r(0)}$ where $f_{e Y}^{\sharp}$ is the $Y$-derivative of $f_{e}^{\sharp}$. Therefore ${ }^{6}$ $\operatorname{Disc}_{Y}\left(f_{e}^{\sharp}\right)=S^{r(0) q\langle 2 m-2\rangle}$ where $\operatorname{Disc}_{Y}\left(f_{e}^{\sharp}\right)$ is the $Y$-discriminant of $f_{e}^{\sharp}$, and hence the Galois group $\operatorname{Gal}\left(f_{e}^{\sharp}, k_{p}\left(X, S, T_{1}, \ldots, T_{e}\right)\right)$ is well-defined as a subgroup of the symmetric group $\operatorname{Sym}_{\langle 2 m-1\rangle}$, and the equation $f_{e}^{\sharp}=0$ gives an unramified covering of the once punctured affine line over $k_{p}\left(X, T_{1}, \ldots, T_{e}\right)$. Since $f_{e}^{\sharp}$ is linear in $X$, by the Gauss Lemma it follows that $f_{e}^{\sharp}$ is irreducible in $k_{p}\left(X, S, T_{1}, \ldots, T_{e}\right)[Y]$, and

\footnotetext{
${ }^{6}$ See the formulas on page 104 of [A03]. As a misprint correction, in line 13 of page 2979 of [A06] $\operatorname{Disc}_{Y}(\phi)=\operatorname{Disc}_{Y}\left(\phi_{e}\right)=1$ should be changed to $\operatorname{Disc}_{Y}(\phi)=\operatorname{Disc}_{Y}\left(\phi_{e}\right)=(-1)^{q^{2 m}}-1$.
} 
hence its Galois group is transitive. Let $\phi_{e}^{\sharp}$ and $\widehat{\phi}_{e}^{\sharp}$ be obtained by putting $T_{m}=1$ and $T_{i}=0$ for $e<i<m$ in $\phi^{b}$ and $\widehat{\phi}^{b}$ respectively, and note that then

$$
\begin{aligned}
\phi_{e}^{\sharp}=\phi_{e}^{\sharp}(Y)=f_{e}^{\sharp}\left(Y^{q-1}\right)= & Y^{q^{2 m}-1}+S^{r(0)}+S^{r(m)} X Y^{q^{m}-1} \\
& +\sum_{i=1}^{e}\left(S^{r(m+i)} T_{i}^{q^{i}} Y^{q^{m+i}-1}+S^{r(m-i)} T_{i} Y^{q^{m-i}-1}\right)
\end{aligned}
$$

and

$$
\begin{aligned}
\widehat{\phi}_{e}^{\sharp}=\widehat{\phi}_{e}^{\sharp}(Y)=Y \phi_{e}^{\sharp}(Y)= & Y^{q^{2 m}}+S^{r(0)} Y+S^{r(m)} X Y^{q^{m}} \\
& +\sum_{i=1}^{e}\left(S^{r(m+i)} T_{i}^{q^{i}} Y^{q^{m+i}}+S^{r(m-i)} T_{i} Y^{q^{m-i}}\right)
\end{aligned}
$$

are the subvectorial and vectorial associates of $f_{e}^{\sharp}$ respectively. By a similar calculation, $\operatorname{Disc}_{Y}\left(\phi_{e}^{\sharp}\right)=(-1)^{q^{2 m}-1} S^{r(0)\left(q^{2 m}-2\right)}$ and $\operatorname{Disc}_{Y}\left(\widehat{\phi}_{e}^{\sharp}\right)=S^{r(0) q^{2 m}}$, and hence the Galois groups $\operatorname{Gal}\left(\phi_{e}^{\sharp}, k_{p}\left(X, S, T_{1}, \ldots, T_{e}\right)\right)$ and $\operatorname{Gal}\left(\widehat{\phi}_{e}^{\sharp}, k_{p}\left(X, S, T_{1}, \ldots, T_{e}\right)\right)$ are well-defined as subgroups of the symmetric groups on $q^{2 m}-1$ and $q^{2 m}$ letters respectively, and the equations $\phi_{e}^{\sharp}=0$ and $\widehat{\phi}_{e}^{\sharp}=0$ give unramified coverings of the once punctured affine line over $k_{p}\left(X, T_{1}, \ldots, T_{e}\right)$. For every divisor $d$ of $q-1$, let $f_{e}^{(d)}, \phi_{e}^{(d)}, \widehat{\phi}_{e}^{(d)}$ be obtained by substituting $S^{d}$ for $S$ in $f_{e}^{\sharp}, \phi_{e}^{\sharp}, \widehat{\phi}_{e}^{\sharp}$ respectively and note that then, as above, the Galois group $\operatorname{Gal}\left(f_{e}^{(d)}, k_{p}\left(X, S, T_{1}, \ldots, T_{e}\right)\right)$ is a well-defined transitive subgroup of $\operatorname{Sym}_{\langle 2 m-1\rangle}$, the Galois groups

$$
\operatorname{Gal}\left(\phi_{e}^{(d)}, k_{p}\left(X, S, T_{1}, \ldots, T_{e}\right)\right) \text { and } \operatorname{Gal}\left(\widehat{\phi}_{e}^{(d)}, k_{p}\left(X, S, T_{1}, \ldots, T_{e}\right)\right)
$$

are well-defined subgroups of the symmetric groups on $q^{2 m}-1$ and $q^{2 m}$ letters respectively, and the equations $f_{e}^{(d)}=0, \phi_{e}^{(d)}=0, \widehat{\phi}_{e}^{(d)}=0$ give unramified coverings of the once punctured affine line over $k_{p}\left(X, T_{1}, \ldots, T_{e}\right)$.

For $0 \leq e \leq m-1$, let $\phi_{e}$ and $f_{e}$ be obtained by putting $S=1$ in $\phi_{e}^{\sharp}$ and $f_{e}^{\sharp}$ respectively. Note that, for $1 \leq e \leq m-1$, these $\phi_{e}$ and $f_{e}$ are the same as those considered in [A06], and if $m>1$, then $\phi_{m-1}$ and $f_{m-1}$ respectively coincide with $\phi$ and $f$ of [A06]. For $0 \leq e \leq m-1$, let $K_{e}=k_{p}\left(X, T_{1}, \ldots, T_{e}\right)$, let $G_{e}$ and $P G_{e}$ be the Galois groups of $\phi_{e}$ and $f_{e}$ over $K_{e}$ respectively, and let $G_{e}^{\sharp}$ and $P G_{e}^{\sharp}$ be the Galois groups of $\phi_{e}^{\sharp}$ and $f_{e}^{\sharp}$ over $K_{e}(S)$ respectively. Likewise, for $0 \leq e \leq m-1$, and for every divisor $d$ of $q-1$, let $G_{e}^{(d)}$ and $P G_{e}^{(d)}$ be the Galois groups of $\phi_{e}^{(d)}$ and $f_{e}^{(d)}$ over $K_{e}(S)$ respectively.

In Section 3, we apply the Mantra of [A08] to the twisted derivative of $f^{b}$ and thereby we prove the Symplectic Rank Theorem (3.6) which says that $P G_{e}^{\sharp}$ is a Rank 3 group with subdegrees $1, q\langle 2 m-3\rangle$ and $q^{2 m-1}$. By again applying the Mantra of [A08] in the Root Extraction Theorem (3.12) of Section 3 we show that, for $0 \leq e \leq$ $m-1$, the splitting field of $\widehat{\phi}_{e}^{\sharp}$ over $K_{e}(S)$ contains a $(q-1)$-th root of its modified constant term $S^{t q^{m}}$; this is an analogue of the $(q-1)$-th root extraction trick given in (2.5)(iii) of [A04] which was used there to go from an SL (= special linear group) covering to a GL (= general linear group) covering. In Section 4, from Theorems (3.6) and (3.12) we deduce Theorem (4.2), which says that if $m>2$ and $\operatorname{GF}(q) \subset k_{p}$ and $\operatorname{GCD}(t, q-1)=1$, then, for $1 \leq e \leq m-1$ and for every divisor $d$ of $q-1$, in a natural manner we have $\operatorname{Sp}(2 m, q)=G_{e} \triangleleft \operatorname{GSp}^{(d)}(2 m, q)=G_{e}^{(d)} \triangleleft G_{e}^{\sharp}=\operatorname{GSp}(2 m, q)$ 
and $\operatorname{PSp}(2 m, q)=P G_{e} \triangleleft \operatorname{PGSp}^{(d)}(2 m, q)=P G_{e}^{(d)} \triangleleft P G_{e}^{\sharp}=\operatorname{PGSp}(2 m, q)$. Note that

$$
\left\{\begin{array}{l}
\text { if } r(i)=\langle 2 m-1\rangle-\langle i-1\rangle \text { for } 0 \leq i \leq 2 m \\
\text { then conditions }(*) \text { and }\left({ }^{* *}\right) \text { are satisfied with } t=q^{m}+1
\end{array}\right.
$$

and

$$
\left\{\begin{array}{l}
\text { if } r(m+i)=0 \text { and } r(m-i)=q^{m-i}\langle i-1\rangle \text { for } 0 \leq i \leq m, \\
\text { then conditions }(*) \text { and }(* *) \text { are satisfied with } t=1 .
\end{array}\right.
$$

Case $\left({ }^{\prime}\right)$ arises when we homogenize $f_{e}$, i.e., when we put $f_{e}^{\sharp}(Y)=S^{\langle 2 m-1\rangle} f_{e}(Y / S)$, and so we may call it the homogeneous case; we shall deal with this in greater detail elsewhere. By analogy, case (") may be called the twisted homogeneous case. In the twisted homogeneous case (") , if $m>2$ and $\operatorname{GF}(q) \subset k_{p}$, then by taking $k=k_{p}$ and $T_{1}=T$ in $\phi_{1}^{\sharp}$ and $f_{1}^{\sharp}$ we get $\Phi^{\sharp}$ and $F^{\sharp}$ respectively, and hence by the above result we have $\operatorname{Gal}\left(\Phi^{\sharp}, k(X, S, T)\right)=\operatorname{GSp}(2 m, q)$ and $\operatorname{Gal}\left(F^{\sharp}, k(X, S, T)\right)=\operatorname{PGSp}(2 m, q)$, and for every divisor $d$ of $q-1$ we have $\operatorname{Gal}\left(\Phi^{(d)}, k(X, S, T)\right)=\mathrm{GSp}^{(d)}(2 m, q)$ and $\operatorname{Gal}\left(F^{(d)}, k(X, S, T)\right)=\operatorname{PGSp}^{(d)}(2 m, q)$. By applying the above result to case $\left(^{\prime \prime}\right)$ we also see that if $m>2$ and $\operatorname{GF}(q) \subset k_{p}$, then for $1 \leq e \leq m-1$ we have $G_{e}=$ $\operatorname{Sp}(2 m, q)$ and $P G_{e}=\operatorname{PSp}(2 m, q)$ which shows that the results of [A06] remain valid without assuming $k_{p}$ algebraically closed.

\section{TWISTED DERIVATIVE AND ITS FACTORIZATION}

Solving the equation $f^{b}=0$ we get

$$
S^{r(m)} X=\frac{\sum_{i=1}^{m}\left(S^{r(m+i)} T_{i}^{q^{i}} Y^{\langle m-1+i\rangle}+S^{r(m-i)} T_{i} Y^{\langle m-1-i\rangle}\right)}{-Y^{\langle m-1\rangle}}
$$

and substituting this in $\frac{f^{\mathrm{b}}(Z)-f^{\mathrm{b}}(Y)}{Z-Y}$ we get

$$
\begin{aligned}
f^{\prime b}(Y, Z)= & \left.\frac{f^{b}(Z)-f^{b}(Y)}{Z-Y} \quad \text { (def of the twisted derivative } f^{\prime b} \text { of } f^{b}\right) \\
= & \frac{\sum_{i=1}^{m}\left(S^{r(m+i)} T_{i}^{q^{i}} Y^{\langle m-1+i\rangle}+S^{r(m-i)} T_{i} Y^{\langle m-1-i\rangle}\right)}{-Y^{\langle m-1\rangle}} \\
& \times \frac{Z^{\langle m-1\rangle}-Y^{\langle m-1\rangle}}{Z-Y} \\
& +\sum_{i=1}^{m}\left(S^{r(m+i)} T_{i}^{q^{i}} \frac{Z^{\langle m-1+i\rangle}-Y^{\langle m-1+i\rangle}}{Z-Y}\right. \\
& \left.+S^{r(m-i)} T_{i} \frac{Z^{\langle m-1-i\rangle}-Y^{\langle m-1-i\rangle}}{Z-Y}\right)
\end{aligned}
$$


and therefore

$g^{\mathrm{b}}=g^{\mathrm{b}}(Y, Z)=Y^{\langle 2 m-1\rangle-1} f^{\prime b}(1 / Y, Z / Y) \quad\left(\right.$ def of polynomial $g^{\mathrm{b}}$ obtained

by dividing roots of $f^{\prime}$ by $Y$ and then changing $Y$ to $1 / Y$ )

$$
\begin{aligned}
= & \sum_{i=1}^{m} S^{r(m+i)} T_{i}^{q^{i}}\left(\frac{Z^{\langle m-1+i\rangle}-1}{Z-1}-\frac{Z^{\langle m-1\rangle}-1}{Z-1}\right) Y^{\langle 2 m-1\rangle-\langle m-1+i\rangle} \\
& -\sum_{i=1}^{m} S^{r(m-i)} T_{i}\left(\frac{Z^{\langle m-1\rangle}-1}{Z-1}-\frac{Z^{\langle m-1-i\rangle}-1}{Z-1}\right) Y^{\langle 2 m-1\rangle-\langle m-1-i\rangle} .
\end{aligned}
$$

By simplifying $g^{b}$ we get

$$
\begin{aligned}
& g^{b}=\sum_{i=1}^{m} S^{r(m+i)} T_{i}^{q^{i}}\left(\frac{Z^{\langle m-1+i\rangle}-Z^{\langle m-1\rangle}}{Z-1}\right) Y^{\langle 2 m-1\rangle-\langle m-1+i\rangle} \\
& -\sum_{i=1}^{m} S^{r(m-i)} T_{i}\left(\frac{Z^{\langle m-1\rangle}-Z^{\langle m-1-i\rangle}}{Z-1}\right) Y^{\langle 2 m-1\rangle-\langle m-1-i\rangle}
\end{aligned}
$$

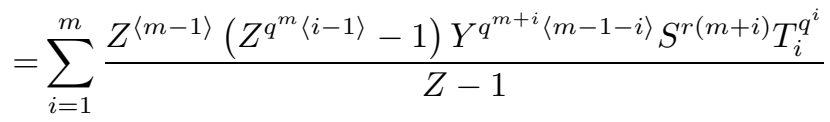

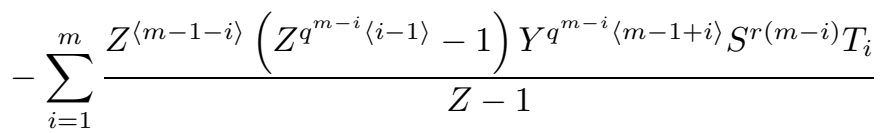

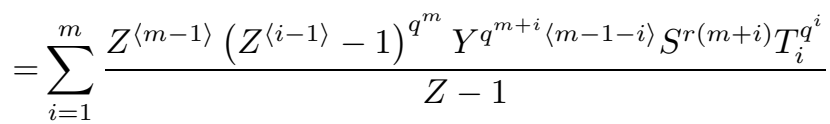

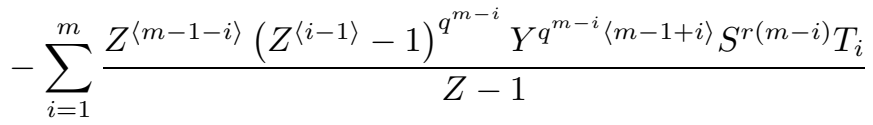

$$
\begin{aligned}
& =\sum_{i=1}^{m} \frac{G_{i}^{q^{i}\langle m-1-i\rangle} H_{i}^{q^{i}}\left(Z(Z-1)^{q-1}\right)^{\langle i-1\rangle} Y^{q^{m}\langle m-1+i\rangle} S^{r(m+i)} T_{i}^{q^{i}}}{Y^{\left(1+q^{m}\right) q^{m}\langle i-1\rangle}} \\
& -\sum_{i=1}^{m} G_{i}^{\langle m-1-i\rangle} H_{i} Y^{q^{m-i}\langle m-1+i\rangle} S^{r(m-i)} T_{i}
\end{aligned}
$$

where for $1 \leq i \leq m$ we have

$G_{i}=Z\left(Z^{\langle i-1\rangle}-1\right)^{q-1} \quad$ and $\quad H_{i}=\frac{Z^{\langle i-1\rangle}-1}{Z-1}=1+Z+Z^{2}+\cdots+Z^{\langle i-1\rangle-1}$.

Hence in view of $(* *)$ we see that

$$
g^{b}=\sum_{i=1}^{m}\left(A^{*\langle i-1\rangle} B_{i}^{* q^{i}}-B_{i}^{*}\right) \quad \text { where } \quad A^{*}=\frac{Z(Z-1)^{q-1}}{\left(Y^{q^{m}+1} S^{t}\right)^{q^{m}}}
$$

and for $1 \leq i \leq m$ we have

$$
B_{i}^{*}=G_{i}^{\langle m-1-i\rangle} H_{i} Y^{q^{m-i}\langle m-1+i\rangle} S^{r(m-i)} T_{i}
$$


and therefore by the Mantra on page 19 of [A08] we get

$$
g^{b}=A^{*} \Gamma^{* q}-\Gamma^{*}=\Gamma^{*}\left(A^{*} \Gamma^{* q-1}-1\right) \quad \text { where } \quad \Gamma^{*}=\sum_{i=1}^{m} \sum_{j=0}^{i-1} A^{*\langle j-1\rangle} B_{i}^{* q^{j}} .
$$

Now, for $0 \leq j<i \leq m$, we clearly have

$$
G_{i}^{q^{j}\langle m-1-i\rangle} H_{i}^{q^{j}}\left(Z(Z-1)^{q-1}\right)^{\langle j-1\rangle}=G_{i}^{\langle m-1-i+j\rangle} H_{i} .
$$

Hence upon letting

$$
g^{\prime b}=\frac{\Gamma^{*}}{\left(Y^{q^{m}+1} S^{t}\right)^{q^{m-1}}}
$$

we get

$$
g^{\prime b}=\sum_{i=1}^{m} \sum_{j=0}^{i-1} G_{i}^{\langle m-1-i+j\rangle} H_{i} Y^{a(i, j)} S^{b(i, j)} T_{i}^{q^{j}}
$$

where, for $0 \leq j<i \leq m$, the integers $a(i, j)$ and $b(i, j)$ are given by

$$
\left\{\begin{array}{l}
a(i, j)=q^{m+j}\langle m-2-j\rangle+q^{m-i+j}\langle i-j-2\rangle \\
\text { and } \\
b(i, j)=q^{j} r(m-i)-t q^{m}\langle j-1\rangle-t q^{m-1}
\end{array}\right.
$$

and out of these $a(i, j)$ is obviously nonnegative and $b(i, j)$ is also nonnegative because by $(* *)$ we have

$$
b(i, j)=q^{j-i} r(m+i)+t q^{m+j-i}\langle i-j-2\rangle \geq 0 .
$$

It follows that

$$
g^{\prime b} \in \operatorname{GF}(p)\left[Z, Y, S, T_{1}, \ldots, T_{m}\right]
$$

and hence upon letting

$$
g^{\prime \prime b}=Z(Z-1)^{q-1}\left(g^{\prime b}\right)^{q-1}-Y^{\left(q^{m}+1\right) q^{m-1}} S^{t q^{m-1}}
$$

we have

$$
g^{\prime \prime b} \in \mathrm{GF}(p)\left[Z, Y, S, T_{1}, \ldots, T_{m}\right]
$$

and, in view of the defining equations of $A$ and $g^{\prime b}$, by the factorization $g^{b}=$ $\Gamma\left(A \Gamma^{q-1}-1\right)$ we get the factorization

$$
g^{b}=g^{\prime b} g^{\prime \prime b} .
$$

By the definitions of $G_{i}$ and $H_{i}$ we see that

$$
\operatorname{deg}_{Z} G_{m}^{\langle m-2\rangle} H_{m}=q\langle 2 m-3\rangle>q\langle m-2+j\rangle=\operatorname{deg}_{Z} G_{i}^{\langle m-1-i+j\rangle} H_{i}
$$

for $0 \leq j<i \leq m$ with $(i, j) \neq(m, m-1)$, and also $a(m, m-1)=0$, and by $\left(^{*}\right)$ and $(* *)$ we have $b(m, m-1)=0$, and hence by the double summation expression for $g^{\prime b}$ we see that $g^{\prime b}$ is a polynomial of degree $q\langle 2 m-3\rangle$ in $Z$ with coefficients in $\operatorname{GF}(p)\left[Y, S, T_{1}, \ldots, T_{m}\right]$ and in it the coefficient of the highest $Z$-degree term is $T_{m}^{q^{m-1}}$. By the definition of $g^{\prime \prime b}$ it now follows that $g^{\prime \prime b}$ is a polynomial of degree $1+(q-1)+(q-1) q\langle 2 m-3\rangle=q^{2 m-1}$ in $Z$ with coefficients in $\operatorname{GF}(p)\left[Y, S, T_{1}, \ldots, T_{m}\right]$ and in it the coefficient of the highest $Z$-degree term is $T_{m}^{(q-1) q^{m-1}}$. Now, upon 
letting $g_{e}^{\sharp}, g_{e}^{\prime \sharp}, g_{e}^{\prime \prime \sharp}$ be obtained by putting $T_{m}=1$ and $T_{i}=0$ for $e<i<m$ in $g^{b}, g^{\prime b}, g^{\prime \prime b}$ respectively, we see that

$$
\left\{\begin{array}{l}
\text { for } 0 \leq e \leq m-1 \text { we have } \\
g_{e}^{\sharp}=g_{e}^{\prime \sharp} g_{e}^{\prime \prime \sharp} \text { where } g_{e}^{\prime \sharp} \text { and } g_{e}^{\prime \prime \sharp} \text { are monic polynomials } \\
\text { of degrees } q\langle 2 m-3\rangle \text { and } q^{2 m-1} \text { in } Z \\
\text { with coefficients in } \operatorname{GF}(p)\left[Y, S, T_{1}, \ldots, T_{e}\right] .
\end{array}\right.
$$

By uniqueness the above factorizations must match with the factorization obtained in [A06]. ${ }^{7}$ To get an explicit match, by splitting the first summation in the expression of $g^{\prime b}$ into two pieces $1 \leq i \leq m-1$ and $i=m$, and then putting $T_{i}=0$ for $e<i<m$ in the first piece and putting $T_{m}=1$ and $j=m-1-\mu$ in the negative of the second piece, we see that

$$
\left\{\begin{array}{l}
\text { for } 0 \leq e \leq m-1 \text { we have } \\
g_{e}^{\prime \sharp}=E_{e}^{\prime}-N^{\prime} \\
\text { where } E_{e}^{\prime}=\sum_{i=1}^{e} \sum_{j=0}^{i-1} G_{i}^{\langle m-1-i+j\rangle} H_{i} Y^{a(i, j)} S^{b(i, j)} T_{i}^{q^{j}} \\
\text { and } N^{\prime}=-\sum_{\mu=0}^{m-1} G_{m}^{\langle m-2-\mu\rangle} H_{m} Y^{\left(q^{m}+1\right) q^{m-1-\mu}\langle\mu-1\rangle} S^{b(m, m-1-\mu)} .
\end{array}\right.
$$

Substituting $g_{e}^{\prime \prime \sharp}$ and $g_{e}^{\prime \sharp}$ for $g^{\prime \prime b}$ and $g^{\prime b}$ in the defining equation of $g^{\prime \prime b}$ we see that

$$
g_{e}^{\prime \prime \sharp}=Z\left((Z-1) g_{e}^{\prime \sharp}\right)^{q-1}-Y^{\left(q^{m}+1\right) q^{m-1}} S^{t q^{m-1}} .
$$

Upon letting $E_{e}^{\prime \prime}=(Z-1) E_{e}^{\prime}$ and $N^{\prime \prime}=(Z-1) N^{\prime} /\left(Z^{\langle m-1\rangle}-1\right)$, by the first equation in (3.3) we get $(Z-1) g_{e}^{\prime \sharp}=E_{e}^{\prime \prime}-\left(Z^{\langle m-1\rangle}-1\right) N^{\prime \prime}$, and hence by the above equation for $g^{\prime \prime \prime}$ we see that

$$
g_{e}^{\prime \prime \sharp}=Z\left(E_{e}^{\prime \prime}-\left(Z^{\langle m-1\rangle}-1\right) N^{\prime \prime}\right)^{q-1}-Y^{\left(q^{m}+1\right) q^{m-1}} S^{t q^{m-1}} .
$$

Using the geometric series identity

$$
(X-Y)^{q-1}=\left(X^{q}-Y^{q}\right) /(X-Y)=\sum_{l=1}^{q} Y^{l-1} X^{q-l}
$$

with $X=E_{e}^{\prime \prime}$ and $Y=\left(Z^{\langle m-1\rangle}-1\right) N^{\prime \prime}$, by the above equation for $g_{e}^{\prime \prime \sharp}$ and the equations for $E_{e}^{\prime}$ and $N^{\prime}$ given in (3.3) we see that

$$
\left\{\begin{array}{l}
\text { for } 0 \leq e \leq m-1 \text { we have } \\
g^{\prime \prime \sharp}=\left(\sum_{l=1}^{q} Z\left(Z^{\langle m-1\rangle}-1\right)^{l-1} N^{\prime \prime l-1} E_{e}^{\prime \prime q-l}\right)-Y^{\left(q^{m}+1\right) q^{m-1}} S^{t q^{m-1}} \\
\text { where } E_{e}^{\prime \prime}=\sum_{i=1}^{e} \sum_{j=0}^{i-1} G_{i}^{\langle m-1-i+j\rangle}\left(Z^{\langle i-1\rangle}-1\right) Y^{a(i, j)} S^{b(i, j)} T_{i}^{q^{j}} \\
\text { and } N^{\prime \prime}=-\sum_{\mu=0}^{m-1} G_{m}^{\langle m-2-\mu\rangle} Y^{\left(q^{m}+1\right) q^{m-1-\mu}\langle\mu-1\rangle} S^{b(m, m-1-\mu)} .
\end{array}\right.
$$

If $m>1$, then the values of $g^{\prime}$ and $g^{\prime \prime}$ given in (3.2) to (3.6) of [A06] visibly coincide with the values obtained by putting $e=m-1$ and $S=1$ in $g_{e}^{\prime \sharp}$ and $g_{e}^{\prime \prime \sharp}$ respectively. Since, for $1 \leq e \leq m-1$, the polynomials $g_{e}^{\prime}$ and $g_{e}^{\prime \prime}$ of [A06] were obtained by putting $T_{i}=0$ for $e<i<m$ in the polynomials $g^{\prime}$ and $g^{\prime \prime}$ respectively, it follows that $g_{e}^{\prime}$ and $g_{e}^{\prime \prime}$ can also be obtained by putting $S=1$ in $g_{e}^{\prime \sharp}$ and $g_{e}^{\prime \prime \sharp}$ respectively.

${ }^{7}$ As a misprint correction, in (3.3) on page 2985 of [A06] the exponent of $\left(Z^{\langle m-1\rangle}-1\right)$ should be changed from $q-1$ to $l-1$, and the exponent of $Y$ should be changed from $\left(q^{m}+1\right)\left(q^{m-1}-1\right)$ to $\left(q^{m}+1\right) q^{m-1}$. 
Therefore by the irreducibility of $g_{e}^{\prime}$ and $g_{e}^{\prime \prime}$ proved in (4.5) of [A06] we conclude that

$$
\left\{\begin{array}{l}
\text { for } 1 \leq e \leq m-1, \\
\text { the polynomials } g_{e}^{\prime \sharp} \text { and } g_{e}^{\prime \prime \sharp} \text { are irreducible in } k_{p}\left(Y, S, T_{1}, \ldots, T_{e}\right)[Z] .
\end{array}\right.
$$

For $1 \leq e \leq m-1$, as we have noted, $f_{e}^{\sharp}$ is irreducible in $K_{e}(S)[Y]$ where $K_{e}=k_{p}\left(X, T_{1}, \ldots, T_{e}\right)$, its twisted derivative is $f_{e}^{\prime \sharp}(Y, Z)$, and $g_{e}^{\sharp}$ is obtained by dividing the $Z$-roots of $f_{e}^{\prime \sharp}(Y, Z)$ by $Y$ and then changing $Y$ to $1 / Y$; therefore by (3.2) and (3.5) we get the following:

Symplectic Rank Theorem (3.6). For $1 \leq e \leq m-1$, the Galois group $P G_{e}^{\sharp}$ of $f_{e}^{\sharp}$ over $K_{e}(S)$ is a transitive permutation group of Rank 3 with subdegrees 1 , $q\langle 2 m-3\rangle$ and $q^{2 m-1}$.

In view of Proposition (3.1) of [A04] we get the following:

Theorem (3.7). If $\operatorname{GF}(q) \subset k_{p}$, then, for $0 \leq e \leq m-1$, for the respective Galois groups $G_{e}^{\sharp}$ and $P G_{e}^{\sharp}$ of $\phi_{e}^{\sharp}$ and $f_{e}^{\sharp}$ over $K_{e}(S)$, in a natural manner we have $G_{e}^{\sharp}<\operatorname{GL}(2 m, q)$ and $\Theta_{2 m}\left(G_{e}^{\sharp}\right)=P G_{e}^{\sharp}<\operatorname{PGL}(2 m, q)$ where $\Theta_{2 m}$ is the canonical epimorphism of $\mathrm{GL}(2 m, q)$ onto $\operatorname{PGL}(2 m, q)$.

Recall that

$$
\widehat{\phi}^{b}(Y)=S^{r(m)} X Y^{q^{m}}+\sum_{i=1}^{m}\left(S^{r(m+i)} T_{i}^{q^{i}} Y^{q^{m+i}}+S^{r(m-i)} T_{i} Y^{q^{m-i}}\right)
$$

is the vectorial associate of $f^{b}(Y)$, and let

$$
\psi^{b}(Y, Z)=Y^{q^{m}} \widehat{\phi}^{b}(Z)-Z^{q^{m}} \widehat{\phi}^{b}(Y) .
$$

Then in view of $(* *)$ we see that

$$
\psi^{b}(Y, Z)=\sum_{i=1}^{m}\left(A^{\mathrm{b}\langle i-1\rangle} B_{i}^{\mathrm{b}}(Y, Z)^{q^{i}}-B_{i}^{\mathrm{b}}(Y, Z)\right) \quad \text { where } \quad A^{\mathrm{b}}=\frac{1}{S^{t q^{m}}}
$$

and for $1 \leq i \leq m$ we have

$$
B_{i}^{\mathrm{b}}(Y, Z)=\left(Z^{q^{m}} Y^{q^{m-i}}-Y^{q^{m}} Z^{q^{m-i}}\right) S^{r(m-i)} T_{i} .
$$

Therefore again by the Mantra on page 19 of [A08] we get

$\psi^{b}(Y, Z)=A^{b} \Gamma^{b}(Y, Z)^{q}-\Gamma^{b}(Y, Z) \quad$ where $\quad \Gamma^{b}(Y, Z)=\sum_{i=1}^{m} \sum_{j=0}^{i-1} A^{b\langle j-1\rangle} B_{i}^{b}(Y, Z)^{q^{j}}$.

Substituting the values of $A^{b}$ and $B_{i}^{b}$ in the defining equation for $\Gamma^{b}$ we get

$$
\Gamma^{b}(Y, Z)=\sum_{i=1}^{m} \sum_{j=0}^{i-1}\left(Z^{q^{m+j}} Y^{q^{m-i+j}}-Y^{q^{m+j}} Z^{q^{m-i+j}}\right) S^{b(i, j)+t q^{m-1}} T_{i}^{q^{j}}
$$

and hence we see that $\Gamma^{b}$ is a polynomial of degree $q^{2 m-1}$ in $Z$ with coefficients in $\operatorname{GF}(p)\left[Y, S, T_{1}, \ldots, T_{m}\right]$ and in it the coefficient of the highest $Z$-degree term is $\left(Y S^{t} T_{m}\right)^{q^{m-1}}$.

Recall that, for $0 \leq e \leq m-1$, the vectorial associate of $f_{e}^{\sharp}(Y)$ is $\widehat{\phi}_{e}^{\sharp}(Y)$ and let

$$
\psi_{e}^{\sharp}(Y, Z)=Y^{q^{m}} \widehat{\phi}_{e}^{\sharp}(Z)-Z^{q^{m}} \widehat{\phi}_{e}^{\sharp}(Y) \text {. }
$$


Then $\psi_{e}^{\sharp}$ can be obtained by putting $T_{m}=1$ and $T_{i}=0$ for $e<i<m$ in the defining equation of $\psi^{\sharp}$, and hence by putting $T_{m}=1$ and $T_{i}=0$ for $e<i<m$ in the above expression of $\psi^{b}$ in terms of $\Gamma^{b}$ we get

$$
\psi_{e}^{\sharp}(Y, Z)=S^{-t q^{m}} \Gamma_{e}^{\sharp}(Y, Z)^{q}-\Gamma_{e}^{\sharp}(Y, Z)
$$

where

$$
\begin{aligned}
\Gamma_{e}^{\sharp}(Y, Z)= & \sum_{i=1}^{e} \sum_{j=0}^{i-1}\left(Z^{q^{m+j}} Y^{q^{m-i+j}}-Y^{q^{m+j}} Z^{q^{m-i+j}}\right) S^{b(i, j)+t q^{m-1}} T_{i}^{q^{j}} \\
& +\sum_{j=0}^{m-1}\left(Z^{q^{m+j}} Y^{q^{j}}-Y^{q^{m+j}} Z^{q^{j}}\right) S^{t q^{j}\langle m-j-1\rangle} .
\end{aligned}
$$

Again, $\Gamma_{e}^{\sharp}$ is obtained by putting $T_{m}=1$ and $T_{i}=0$ for $e<i<m$ in $\Gamma^{b}$, and hence

$$
\left\{\begin{array}{l}
\text { for } 0 \leq e \leq m-1 \text { we have that } \\
\Gamma_{e}^{\sharp} \text { is a polynomial of degree } q^{2 m-1} \text { in } Z \\
\text { with coefficients in } \operatorname{GF}(p)\left[Y, S, T_{1}, \ldots, T_{e}\right] \text { and in it } \\
\text { the coefficient of the highest } Z \text {-degree term is }\left(Y S^{t}\right)^{q^{m-1}} .
\end{array}\right.
$$

For $0 \leq e \leq m-1$, since $\operatorname{deg}_{Y} \widehat{\phi}_{e}^{\sharp}(Y)=q^{2 m}$ and $\operatorname{Disc}_{Y} \widehat{\phi}_{e}^{\sharp}(Y)=S^{r(0) q^{2 m}}$, in view of (3.8), (3.9) and (3.11), we see that there exists a nonzero root $y_{e}$ of $\widehat{\phi}_{e}^{\sharp}(Y)$ in any splitting field $L_{e}^{\sharp}$ of $\widehat{\phi}_{e}^{\sharp}(Y)$ over $K_{e}(S)$ where $K_{e}=k_{p}\left(X, T_{1}, \ldots, T_{e}\right)$, and given any such $y_{e}$ there exists a root $z_{e}$ of $\widehat{\phi}_{e}^{\sharp}(Y)$ in $L_{e}^{\sharp}$ such that $\Gamma_{e}^{\sharp}\left(y_{e}, z_{e}\right) \neq 0$, and for every such $z_{e}$ we have $\Gamma_{e}^{\sharp}\left(y_{e}, z_{e}\right)^{q-1}=S^{t q^{m}}$. Clearly $\operatorname{GCD}\left(t q^{m}, q-1\right)=\operatorname{GCD}(t, q-1)$, and hence for any divisor $d$ of $(q-1) / \operatorname{GCD}(t, q-1)$, we can find integers $\sigma, \tau$ with $\sigma t q^{m}+\tau(q-1)=(q-1) / d$, and for any such roots $y_{e}, z_{e}$ and any such integers $\sigma, \tau$, upon letting $\widehat{\Lambda}_{e}=\Gamma_{e}^{\sharp}\left(y_{e}, z_{e}\right)^{\sigma} S^{\tau}$ we see that $\widehat{\Lambda}_{e}^{q-1}=S^{(q-1) / d}$ with $\widehat{\Lambda}_{e} \in L_{e}^{\sharp}$. If also $\operatorname{GF}(q) \subset k_{p}$, then we can find $\lambda \in \operatorname{GF}(q) \subset k_{p}$ such that upon letting $\Lambda_{e}=\lambda \widehat{\Lambda}_{e}$ we have $\Lambda_{e}^{d}=S$ with $\Lambda_{e} \in L_{e}^{\sharp}$, and now, because $L_{e}^{\sharp}$ is also a splitting field of $\phi_{e}^{\sharp}$ over $K_{e}(S)$, by the Substitution Principle on page 98 of [A03], for the Galois groups $G_{e}^{(d)}$ and $G_{e}^{\sharp}$ of $\phi_{e}^{(d)}$ and $\phi_{e}^{\sharp}$ over $K_{e}(S)$ respectively, in a natural manner we have $G_{e}^{(d)}=\operatorname{Gal}\left(\phi_{e}^{\sharp}, K_{e}\left(\Lambda_{e}\right)\right) \triangleleft G_{e}^{\sharp}$ with $G_{e}^{\sharp} / G_{e}^{(d)}=\operatorname{Gal}\left(K_{e}\left(\Lambda_{e}\right), K_{e}(S)\right)=Z_{d}$.

For $0 \leq e \leq m-1$, let $R=k_{e}\left[X, S, T_{1}, \ldots, T_{e}\right]$ and $\bar{R}=k_{p}\left[X, T_{1}, \ldots, T_{e}\right]$, and let $\alpha: R \rightarrow \bar{R}$ be the unique $\bar{R}$-epimorphism which sends $S$ to 1 . Then $K_{e}(S)$ and $K_{e}$ are the quotient fields of $R$ and $\bar{R}$ respectively, and for every divisor $d$ of $q-1$ we have that $\phi_{e}^{(d)}$ is a monic polynomial in $Y$ with coefficients in $R$, and by applying $\alpha$ to the coefficients of $\phi_{e}^{(d)}$ we get the polynomial $\phi_{e}$ which is such that $\operatorname{Disc}_{Y}\left(\phi_{e}\right) \neq 0$, and therefore, for the Galois group $G_{e}$ of $\phi_{e}$ over $K_{e}$, in a natural manner we have $G_{e}<G_{e}^{(d)}$. $^{8}$

\footnotetext{
${ }^{8}$ Here we are using the Specialization Principle which follows from the material of Section 2 of [A01] and which says that if $U$ is a monic polynomial in $Y$ with coefficients in a normal integral domain $R$ which is a localization of an affine ring over a field or a pseudogeometric Dedekind domain, and if $\alpha: R \rightarrow \bar{R}$ is an epimorphism of $R$ onto an integral domain $\bar{R}$ such that for the polynomial $\bar{U}$ obtained by applying $\alpha$ to the coefficients of $U$ we have $\operatorname{Disc}_{Y}(\bar{U}) \neq 0$, then, in a natural manner, the Galois group of $\bar{U}$ over the quotient field of $\bar{R}$ is isomorphic (as a permutation group) to a subgroup of the Galois group of $U$ over the quotient field of $R$. The Specialization Principle is also true when, instead of the above assumptions on $R$, we assume $R$ to be a UFD; see page 190 of [Wae].
} 
Thus we get the following Theorem which may be considered analogous to part (8) of the Composite Polynomial Lemma (2.4) on pages 13-14 of [A04].

Root Extraction Theorem (3.12). For $0 \leq e \leq m-1$, there exists a nonzero root $y_{e}$ of $\widehat{\phi}_{e}^{\sharp}(Y)$ in any splitting field $L_{e}^{\sharp}$ of $\widehat{\phi}_{e}^{\sharp}(Y)$ over $K_{e}(S)$ where $K_{e}=$ $k_{p}\left(X, T_{1}, \ldots, T_{e}\right)$, and given any such $y_{e}$ there exists a root $z_{e}$ of $\widehat{\phi}_{e}^{\sharp}(Y)$ in $L_{e}^{\sharp}$ such that $\Gamma_{e}^{\sharp}\left(y_{e}, z_{e}\right) \neq 0$, and for every such $z_{e}$ we have $\Gamma_{e}^{\sharp}\left(y_{e}, z_{e}\right)^{q-1}=S^{t^{m}}$ (note that for any $y_{e} \in L_{e}^{\sharp}$ and $z_{e} \in L_{e}^{\sharp}$ we obviously have $\left.\Gamma_{e}^{\sharp}\left(y_{e}, z_{e}\right) \in L_{e}^{\sharp}\right)$. Moreover, for every divisor $d$ of $(q-1) / \operatorname{GCD}(t, q-1)$, there exist integers $\sigma, \tau$ with $\sigma t q^{m}+\tau(q-1)=(q-1) / d$, and if $\operatorname{GF}(q) \subset k_{p}$, then, given any such roots $y_{e}, z_{e}$ and any such integers $\sigma, \tau$, there exists $\lambda \in \mathrm{GF}(q) \subset k_{p}$ such that for $\Lambda_{e}=\lambda \Gamma_{e}^{\sharp}\left(y_{e}, z_{e}\right)^{\sigma} S^{\tau}$ we have $\Lambda_{e} \in L_{e}^{\sharp}$ with $\Lambda_{e}^{d}=S$, and for the respective Galois groups $G_{e}, G_{e}^{(d)}, G_{e}^{\sharp}$ of $\phi_{e}, \phi_{e}^{(d)}, \phi_{e}^{\sharp}$ over $K_{e}, K_{e}(S), K_{e}(S)$ respectively, in a natural manner we have $G_{e}<G_{e}^{(d)} \triangleleft G_{e}^{\sharp}$ with $G_{e}^{\sharp} / G_{e}^{(d)}=Z_{d}$.

\section{Galois groups}

By 2.1.2, 2.1.B and 2.1.C of [KLi] we have

$$
\operatorname{Sp}(2 m, q) \triangleleft \operatorname{GSp}(2 m, q) \quad \text { with } \quad \operatorname{GSp}(2 m, q) / \operatorname{Sp}(2 m, q)=Z_{q-1}
$$

and hence in view of $(4.6),(4.7),(5.1),(5.6)$ and (5.8) of [A06], by our Theorems (3.6), (3.7) and (3.12) we get the following: ${ }^{9}$

Theorem (4.1). If $m>2$ and $\mathrm{GF}(q) \subset k_{p}$, then, for $1 \leq e \leq m-1$ and for every divisor $d$ of $(q-1) / \operatorname{GCD}(t, q-1)$, in a natural manner we have

$$
\mathrm{Sp}(2 m, q) \triangleleft G_{e} \triangleleft G_{e}^{(d)} \triangleleft G_{e}^{\sharp} \triangleleft \mathrm{GSp}(2 m, q) \quad \text { with } \quad G_{e}^{\sharp} / G_{e}^{(d)}=Z_{d}
$$

and

$$
\mathrm{PSp}(2 m, q) \triangleleft P G_{e} \triangleleft P G_{e}^{(d)} \triangleleft P G_{e}^{\sharp} \triangleleft \mathrm{PGSp}(2 m, q)
$$

where we recall that $G_{e}, G_{e}^{(d)}, G_{e}^{\sharp}, P G_{e}, P G_{e}^{(d)}, P G_{e}^{\sharp}$ are the Galois groups of $\phi_{e}, \phi_{e}^{(d)}$, $\phi_{e}^{\sharp}, f_{e}, f_{e}^{(d)}, f_{e}^{\sharp}$ over $K_{e}, K_{e}(S), K_{e}(S), K_{e}, K_{e}(S), K_{e}(S)$ respectively with $K_{e}=$ $k_{p}\left(X, T_{1}, \ldots, T_{e}\right)$.

In view of (3.7) and (4.0), by taking $d=q-1$ in (4.1) we see that

$\left\{\begin{array}{l}\text { if } m>2 \text { and } \operatorname{GF}(q) \subset k_{p} \text { and } \operatorname{GCD}(t, q-1)=1 \text {, then for } 1 \leq e \leq m-1 \text { we have } \\ \operatorname{Sp}(2 m, q)=G_{e} \triangleleft G_{e}^{\sharp}=\operatorname{GSp}(2 m, q) \text { and } \operatorname{PSp}(2 m, q)=P G_{e} \triangleleft P G_{e}^{\sharp}=\operatorname{PGSp}(2 m, q)\end{array}\right.$ and therefore again by (4.1) we get the following:

Theorem (4.2). If $m>2$ and $\operatorname{GF}(q) \subset k_{p}$ and $\operatorname{GCD}(t, q-1)=1$, then, for $1 \leq e \leq m-1$ and for every divisor $d$ of $(q-1)$, in a natural manner we have

$$
\operatorname{Sp}(2 m, q)=G_{e} \triangleleft \operatorname{GSp}^{(d)}(2 m, q)=G_{e}^{(d)} \triangleleft G_{e}^{\sharp}=\operatorname{GSp}(2 m, q)
$$

and

$$
\operatorname{PSp}(2 m, q)=P G_{e} \triangleleft \operatorname{PGSp}^{(d)}(2 m, q)=P G_{e}^{(d)} \triangleleft P G_{e}^{\sharp}=\operatorname{PGSp}(2 m, q)
$$

\footnotetext{
${ }^{9}$ As a misprint correction, in (5.8) on page 2990 of [A06], $\operatorname{PSp}(2 m, q) \triangleleft \delta^{-1} G \delta$ should be changed to $\operatorname{PSp}(2 m, q) \triangleleft \delta^{-1} G \delta \triangleleft \operatorname{PGSp}(2 m, q)$. As another misprint correction, in (6.1) on page 2990 of [A06], $\operatorname{Gal}\left(\phi, k_{p}\left(X, T_{1}, \ldots, T_{e}\right)\right)$ and $\operatorname{Gal}\left(f, k_{p}\left(X, T_{1}, \ldots, T_{e}\right)\right)$ should be changed to $\operatorname{Gal}\left(\phi, k_{p}\left(X, T_{1}, \ldots, T_{m-1}\right)\right)$ and $\operatorname{Gal}\left(f, k_{p}\left(X, T_{1}, \ldots, T_{m-1}\right)\right)$ respectively.
} 
where we recall that $G_{e}, G_{e}^{(d)}, G_{e}^{\sharp}, P G_{e}, P G_{e}^{(d)}, P G_{e}^{\sharp}$ are the Galois groups of $\phi_{e}, \phi_{e}^{(d)}$, $\phi_{e}^{\sharp}, f_{e}, f_{e}^{(d)}, f_{e}^{\sharp}$ over $K_{e}, K_{e}(S), K_{e}(S), K_{e}, K_{e}(S), K_{e}(S)$ respectively with $K_{e}=$ $k_{p}\left(X, T_{1}, \ldots, T_{e}\right)$.

Remark (4.3). By applying (4.2) to case (" $\left.{ }^{\prime \prime}\right)$ we see that if $m>2$ and $\operatorname{GF}(q) \subset k_{p}$, then, for $1 \leq e \leq m-1$, in a natural manner we have $\operatorname{Gal}\left(\phi_{e}, k_{p}\left(X, T_{1}, \ldots, T_{e}\right)\right)=$ $\operatorname{Sp}(2 m, q)$ and $\operatorname{Gal}\left(f_{e}, k_{p}\left(X, T_{1}, \ldots, T_{e}\right)\right)=\operatorname{PSp}(2 m, q)$, which is an improvement on Theorem (6.2) of [A06] as we no longer need the condition that $k_{p}$ is algebraically closed. We shall discuss the $m \leq 2$ case of (4.2) elsewhere.

\section{REFERENCES}

[A01] S. S. Abhyankar, Local uniformization on algebraic surfaces over ground fields of characteristic $p \neq 0$, Annals of Mathematics 63 (1956), 491-526. MR 17:1134d

[A02] S. S. Abhyankar, Coverings of algebraic curves, American Journal of Mathematics 79 (1957), 825-856. MR 20:872

[A03] S. S. Abhyankar, Galois theory on the line in nonzero characteristic, Bulletin of the American Mathematical Society 27 (1992), 68-133. MR 94a:12004

[A04] S. S. Abhyankar, Nice equations for nice groups, Israel Journal of Mathematics 88 (1994), 1-24. MR 96f: 12003

[A05] S. S. Abhyankar, Again nice equations for nice groups, Proceedings of the American Mathematical Society 124 (1996), 2967-2976. MR 96m:12004

[A06] S. S. Abhyankar, More nice equations for nice groups, Proceedings of the American Mathematical Society 124 (1996), 2977-2991. MR 96m:12005

[A07] S. S. Abhyankar, Further nice equations for nice groups, Transactions of the American Mathematical Society 348 (1996), 1555-1577. MR 96m:14021

[A08] S. S. Abhyankar, Factorizations over finite fields, Finite Fields and Applications, London Mathematical Society, Lecture Note Series 233 (1996), 1-21. CMP 97:08

[A09] S. S. Abhyankar, Projective polynomials, Proceedings of the American Mathematical Society 125 (1997), 1643-1650. CMP 97:07

[BuS] F. Buekenhout and E. E. Shult, On the foundations of polar geometry, Geometriae Dedicata 3 (1974), 155-170. MR 50:3091

[CaK] P. J. Cameron and W. M. Kantor, 2-transitive and antiflag transitive collineation groups of finite projective spaces, Journal of Algebra 60 (1979), 384-422. MR 81c:20032

[Kan] W. M. Kantor, Rank 3 characterizations of classical geometries, Journal of Algebra 36 (1975), 309-313. MR 52:8229

[KLi] P. Kleidman and M. W. Liebeck, The Subgroup Structure of the Finite Classical Groups, Cambridge University Press, Cambridge, 1990. MR 91g:20001

[Tit] J. Tits, Buildings of Spherical Type and Finite BN-Pairs, Springer Lecture Notes In Mathematics Number 386, 1974. MR 57:9866

[Wae] B. L. van der Waerden, Modern Algebra, vol I, Frederick Ungar Publishing Co., New York, 1949. MR 10:587b

Department of Mathematics, Purdue University, West Lafayette, Indiana 47907

E-mail address: ram@cs.purdue.edu

E-mail address: loomisp@math.purdue.edu 